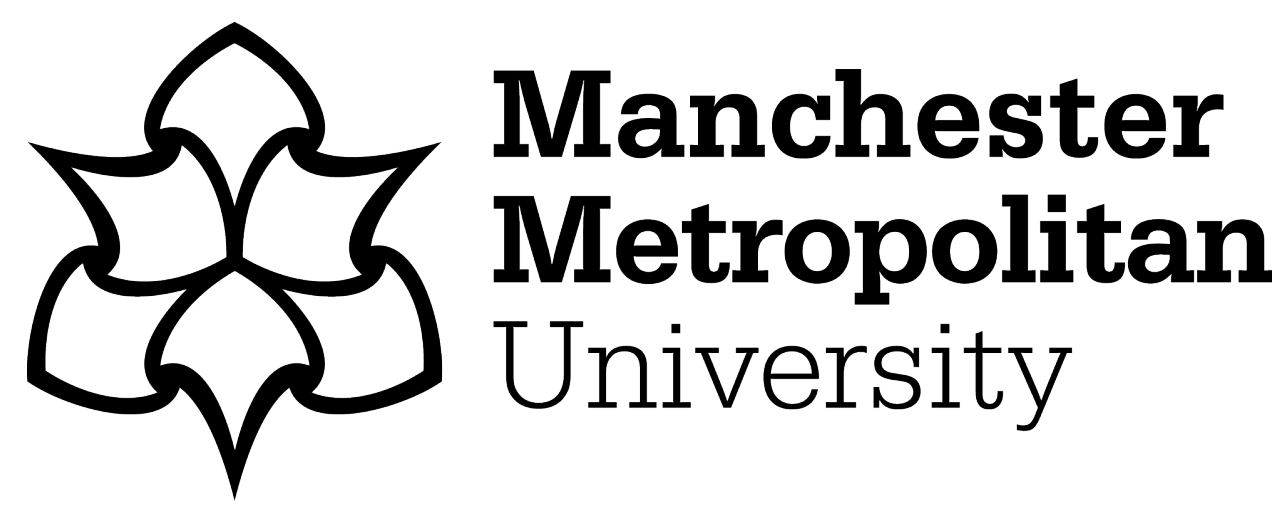

Giorgi, I, Watson, C, Pratt, C and Masala, GL (2020) Designing robot verbal and nonverbal interactions in socially assistive domain for quality ageing in place. In: International KES Conference on Human Centred Intelligent Systems 2020 (KES HCIS 2020), 17 June 2020 - 19 June 2020, Virtual.

Downloaded from: https://e-space.mmu.ac.uk/626173/

Version: Accepted Version

Publisher: Springer

DOI: https://doi.org/10.1007/978-981-15-5784-2_21

Please cite the published version 


\title{
Designing robot verbal and non-verbal interactions in socially assistive domain for quality ageing in place
}

\author{
Ioanna Giorgi ${ }^{10000-0001-9583-6959]}$, Catherine Watson ${ }^{2}$, Cassiana Pratt ${ }^{2}$, Giovanni L. \\ Masala ${ }^{2[0000-0001-6734-9424] ~}$ \\ ${ }^{1}$ The University of Manchester, UK \\ ${ }^{2}$ Manchester Metropolitan University, UK
}

\begin{abstract}
Endowing robots with the role of social assistance in silver care could be a powerful tool to combat chronic loneliness in ageing adults. These robots can be tasked with functional and affective care to support quotidian living and grant companionship that helps lessen the burden of cognitive decline and impairment emerging from social isolation. To accomplish such imperative tasks, artificial agents must be adept at communicating naturally with the human elder. In this work, we aim to enable human-robot interaction by designing human-like verbal and nonverbal behaviours of an autonomous robot companion. We employed the robot on a trial run using customisable algorithms to address a range of needs, while thriving social and emotional attachment with the potential senior user, with the final intent being that such endeavours can help achieve quality ageing in place.
\end{abstract}

Keywords: socially assistive, robot companion, eldercare, aged care, verbal and nonverbal interaction, human-robot interaction, natural communication

\section{Introduction}

Robots are greatly becoming important technical resources. Their ability to understand and communicate in a humanlike way allows them to act like social actors and contribute in assisting humans in their households, communicating directly with smart devices and in turn be understood by humans and their social networks via friendly interactions. Several demographic studies have reported a fast ageing population in advanced economies across the world, often referred to as "the silver tsunami" [1], leading to greater attention in designing artificial companions for social purposes and elderly care. Other studies showed an increasing number of elderlies living alone and although the human lifespan is increasing, for most quality of life is decreasing, as elderly people are suffering from isolation, decreased mobility, impaired vision and hearing, memory loss and several mental health issues [2]. There is a paramount need for technological companions that can help society overcome these obstacles surrounding our elderly neighbours, providing them to age well in their homes autonomously for longer, combat loneliness and perform their everyday life routines. 
While seminal contributions have been made in terms of social robots for aged care, there exist certain significant problems. On one hand, the existing platforms and solutions are yet not fully commercially available or can be accessed at a high cost. Furthermore, most come with pre-programmed services, the range of which is often short to meet the constantly changing needs of elderlies [3]. On the other hand, not much is yet known about what influences the way elderly people perceive social robots and to what extent they welcome them in their homes, in particular robots at an early developmental stage [4].

Most works regarding aged care address elderly residents that suffer from cognitive or physical impairment [5-17], focusing less on the risk of them being isolated and lonely. Via this work, we aim to contribute in constantly improving the quality of life of our senior neighbours via programmable social robot companions by designing simple agent behaviours that account for several requirements and challenges in the elder's everyday life. We propose the use of an autonomous socially assistive robot to naturally interact with the senior end-users and proactively engage them with easy quotidian activities. We trained the agent using simple algorithms that in addition can be further customised and personalised to meet a range of specific needs of the targeted user.

The rest of the paper is organised as follows. In section 2, we briefly review the most relevant contributions in the field of social robotics for eldercare. Section 3 introduces the proposed method that employs the humanoid robot NAO as the agent caregiver, followed by a detailed description and planning of the designed activities. The laboratory-based trials and the estimated behaviour of the autonomous agent are given in section 4. The work is concluded in section 5.

\section{Social Robots for Aged Care}

Advances in robotics research and human-robot interaction have provided significant solutions for important challenges of aging populations, acting as an aid in treatment [5], mental health - dementia [6-9], physical health and therapy [10, 11], social assistants and home companions. Pet-like prototypes like iCat, AIBO, Paro, NeCoRo have proven to be helpful when trialled with the targeted elderly people, in particular those suffering from cognitive impairments, such as dementia. Trials and preliminary results have reported increased level of happiness, pleasure and engagement, whilst significantly fighting the sense of restlessness, agitation and loneliness in older adults [9, 1214]. Social and mental commitment robots have been designed, of which Matilda, an assistive robot able to recognise voices, faces and emotions among users and providing services like dancing, telling the news and weather or even making Skype calls $[8,15]$. Matilda (also called PaPeRo) showed positive engagement in residential senior care [15] and home-based care [16]. Other robots, like Mabu, are designed to aid the elderly with regular intake of medications, learning their use and side effects, remembering dispensing schedules and setting reminders [5].

A core concern when designing artificial companions is the simulation of social interaction. Many of the existing prototypes for aged care lack humanlike attributes, such as human voice, gestures or emotion [4]. Researchers aim to have assistive robots that 
convey compassionate qualities [17], with more studies finding elderly individuals are more likely to interact with a humanlike (more child-like) or animal-resembling agent rather than a screen [17]. Furthermore, robots should be able to learn behavioural patterns on individual patients and adapt to future encounters [6]. To improve the support offered in aged care, humanoid social robots have been designed and broadly used to provide physical and emotional wellbeing of the elderlies, here mentioning NAO, Pepper, Brian 2.1, Hobbit, Bandi, Nexi and Matilda [15, 16, 18-21].

Focusing mainly on the emotional well-being of the elderly, these social assistive robots have shown promising improvements on both cognitive declined older adults that suffer from loneliness or isolation and cognitive impaired patients, in particular residents with dementia. For instance, a research aimed to use Pepper as a companion robot that can autonomously understand when an elderly is in need of attention and willing to start an interaction, and which can answer this need by initiating the conversation. The research targeted loneliness among other things, which is not only experienced when living alone, but also in care homes where it is more challenging to detect, revealing its potential with the agent care able to take the right decision $59 \%$ of the time by the end of the experiments [22]. Positive impacts have been reported from the use of social assistive robots to detect early signs of mild cognitive impairments, by guiding its users to complete cognitive tasks. The prototype developed within the MoveCare project was used to evaluate the acceptability and usability of such agents, with promising results showing how old people were more opened to the guidance of a robot, understanding and comfortably accepting its supervision instead of a clinician's [23]. Other researches using social robots proved that NAO robots can improve the communication among older people [24], Hobbit robot helped the elderly engage more in their daily activities [18], Brian 2.1. robot could assist them in social activities [19] and Kabochan Nodding Communication robot showed satisfactory improvements in executive and memory functions, when used to assist in cognitive activities [25].

\section{Method}

\subsection{The Robotic Caregiver}

The reason why a humanoid robot, like NAO, is preferred over virtual assistants for silver care, is because it provides a powerful tool to combat loneliness, as the interactions are more personable and there is a physical presence with whom the users can create social and emotional attachment. Humanoid robots have many humanlike attributes, such as human voice and gestures and can perform similar activities like walking around, pointing, grasping and so on. Therefore, they can offer more support to their caretakers as they can assist with or conduct tasks for them, especially helpful for the elderly with reduced mobility.

NAO's Specifications. The academic version of the robotic platform features 25 degrees of freedom (DoF), an inertial measurement unit with accelerometer, a gyro meter, 4 directional microphones that enhance the sound source location system, a stereo 
broadcast system equipped with 2 loudspeakers, 2 HD cameras for computer vision, head, hand and foot sensors and bumpers and four ultrasonic sensors that provide NAO with stability and positioning within space [26]. The version used here includes strong metallic joints, improved grip and a speech recognition module with 3 languages (English, French and Japanese). The robot is controlled by a specialised Linux-based OS, dubbed NAOqi [26]. The interface for the communication with the user consists of a graphical programming tool named Choregraphe [26], with the latest release 2.8.5.10 used in this work, a simulation software package and a software developer's kit.

NAO for (Health)Care. The robot is a powerful ambassador in healthcare advocacy, raising disease awareness and helping patients with disease management and have largely become integrated caregivers in healthcare institutions. Typical use cases of NAO in the Healthcare [27] include Health Assistant, aiding patients with self-diagnosis, telemedicine and information distribution (alerts, fall detection); Communicator, in prevention care, community activities with children and elderly, given a multi-language fluency and translation via Cloud Services; Edutainment, supporting people's physical and mental wellbeing through exercise and entertainment, while advocating educational healthcare context; Receptionist, assisting human staff in high-quality welcoming visitors and patients, managing queues, registration and FAQs; Service Providers, such as consultations, generating healthcare reports on-demand; and counting [27].

\subsection{Experimental Design}

To fulfil the aim of this work, we design and test some functionalities of a social robot (NAO) mainly on: (a) disease management, helping the users with the daily intake of their medicines, setting reminders, providing descriptions and side effects of the medicine; (b) entertainment, not only for the purpose of combating loneliness, but also supporting their independent living, by helping them perform basic tasks such as making phone calls, web navigating and other uses of technology in their homes.

The experiment was designed as a one-to-one interaction of the end-user and the robot in a relatively real-life like environment. The human participant role-played the potential elderly that receives care from the robotic agent through a series of activities. The tasks to be performed in each scene (see Fig.1) were divided in three categories:

1. The agent engages with the human in simple verbal interactions and/or in activities with social or healthcare context (singing, exercising) to help combat loneliness.

2. The artificial agent assists the seniors manage their medications, remembering dispensing schedules and setting reminders or helping the less able elder take the right medicine.

3. The agent helps the elderly understand and use different forms of technology in their home, such as making phone calls with their loved ones, web navigating, using their mobiles/tablets/computers and so on. 
Each of the above tasks determined the behaviour of the robotic caregiver and the actions it performed: speech recognition \& generation, face \& object recognition, static movement or moving in the direction of a target (e.g. towards the elderly) and pointing.

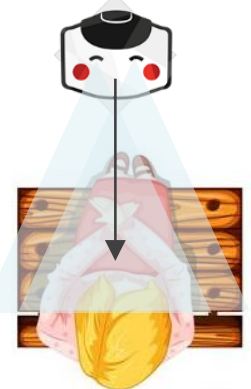

(a)

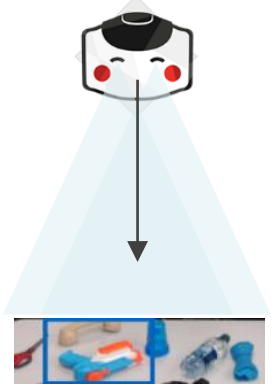

(b)

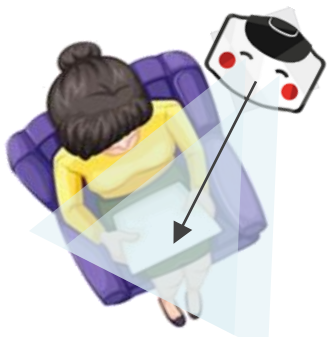

(c)

Fig. 1. Setting of the experimental setup: the participant engages in role-play activities with the robot given three scenarios (a) interaction in the form of verbal conversations or performing an exercise routine, (b) managing regular intake of medications by reminding, describing or pointing at the correct scheduled medicine and, (c) assisting the user access and navigate a Webpage.

Interaction activity. NAO begins the interaction with the elderly owner by calling their attention with a greeting, e.g. "Hello! How are you feeling today?" If the response of the elderly indicates positive feeling, the robot expresses enthusiasm ("That's great!"); otherwise it takes further actions to reverse the situation, by offering them a choice of different options. The elderly can decide if they want to talk about a certain topic ("Do you want to hear a joke?", "Do you want me to tell you a story?"), if they want to exercise or if they prefer to do nothing instead.

Movement. In the event of an exercise request, NAO will ask the elderly to follow along while it performs an easy exercise routine that is beneficial for awakening and stretching their muscles, acknowledging their efforts and encouraging them all along through verbal interaction. The routine can be personalised for the elderly, looked into and researched prior to being performed by the robot, to better serve the physical state and ability of the user. In between the routine, NAO speaks (and listens) to the human, prompting the next moves and asking if they are feeling well. If not, NAO will cease the routine. Interruption can also be triggered if the participant decides they want to stop.

Facial expression recognition. Finally, if the participant chooses the option "Do nothing right now", the robot will consider their decision, however it might monitor their emotional state, through facial expression recognition and can trigger interaction if it detects bad mood (assuming the elderly lingers in the visibility range of NAO). 
Medication management activity. NAO remembers a list of medicine prescriptions, along with their dose intake and the dispensing schedule for each medicine. It sets and monitors reminders to help the elderly regularly take their medication. When a reminder pops up, NAO will query their elder on their medicine intake "Have you taken your medicine today?" Should the answer be negative, the robot will offer further assistance. Given the timeslot, the agent caregiver encourages the elder to take their medicine, with some additional information on the type of medicine, e.g. "Time to take the pill for diabetes" and, asks "Would you like me to help you choose which medicine".

Object recognition \& pointing. Depending on the user's decision, the robot will perform either of the actions: (a) do nothing because they can take the medicine independently, (b) describe the correct medicine box or (c) point the correct medicine box for the elder to grasp. The latter can only be performed assuming the boxes are located in front of the robot (e.g. the robot asks the human to put the boxes in a table in front of it). Regardless of the triggered behaviour, there will always be some sort of verbal interaction (e.g. "Remember to take a glass of water") to convey a sense of care for the elder.

Technology assisting activity: The main designed activities consist on helping the human participant navigate a certain webpage or perform a VoIP call from a messenger like application. NAO is trained with the ability to recognise its elder caretaker and the basic features of a webpage, such as URL/search bar, close button, menu button or the messaging interface, contacts list, call button. The basic principle behind the working mechanism for the Web navigation task is illustrated in the flowchart in Fig. 2

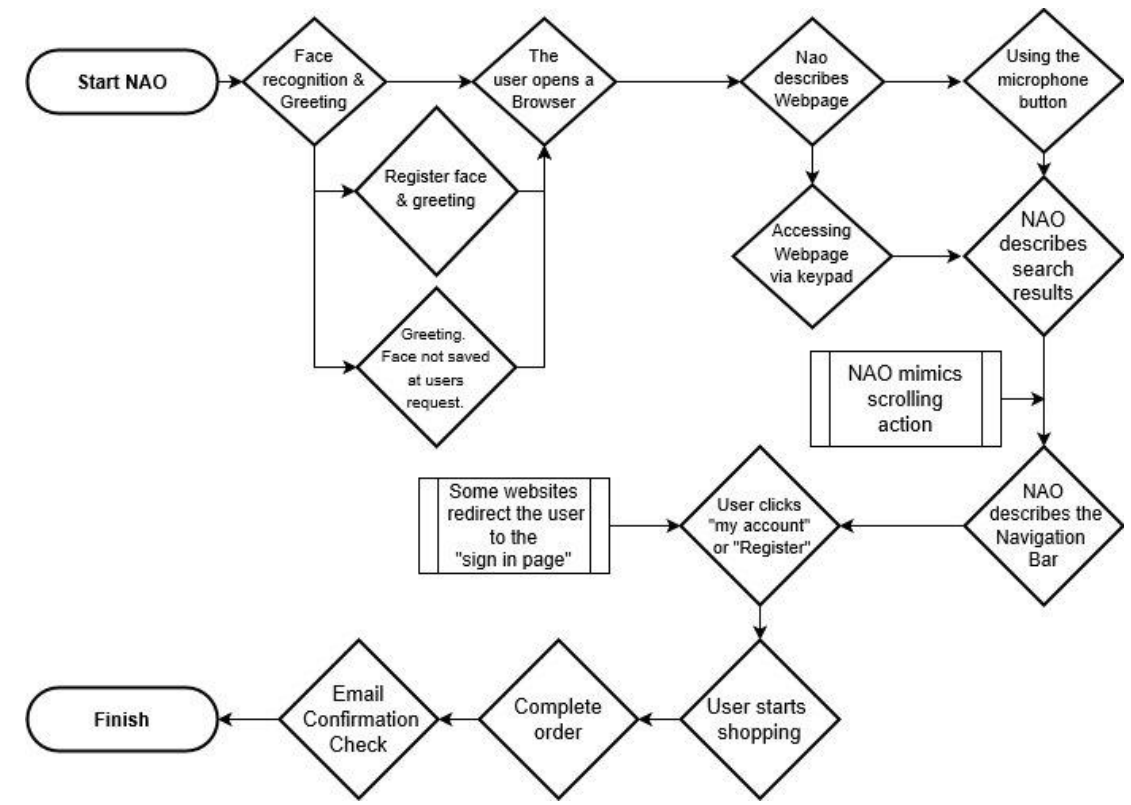

Fig. 2. Flowchart of the working mechanism for the Web navigation task 
Initially, a device is placed in front of NAO, preferably a mobile/tablet or a computer monitor. The robot detects which device is being used. The elder will trigger the agent's behaviour by telling them what they want to do (e.g. "I want to shop some groceries"). For the webpage navigation activity, we selected a supermarket retailer webpage and a retailer webpage for any other shopping purposes. The robot will guide the owner through a step by step process that the elder will perform to locate and open the browser and navigate around the website of choice. The user is taken to login their details into the website to access their account. If they already have an account, they can start shopping. Once the order is complete the user can then check their email for an order confirmation. Throughout the process, the user can ask NAO to go back a step.

\section{Trial runs}

The pilot activities with the NAO robot were implemented using Python programming language and the toolboxes in the Choregraphe interface version 2.8. We carried out live testing experiments with academic NAO V6 in a home-simulated laboratory environment, some of which are illustrated in Fig. 4, Fig. 5 and Fig. 6. We ran each scene independently, trying all possible interactions between the user and the robot, whether the user would engage in a verbal conversation, an exercise routine, receiving help with medication intake or web searching. The final aim was to investigate if the robot could act autonomously in accordance with the expected designed behaviour and assess the potentiality of these agents becoming an integrated part of the elderlies' homes.

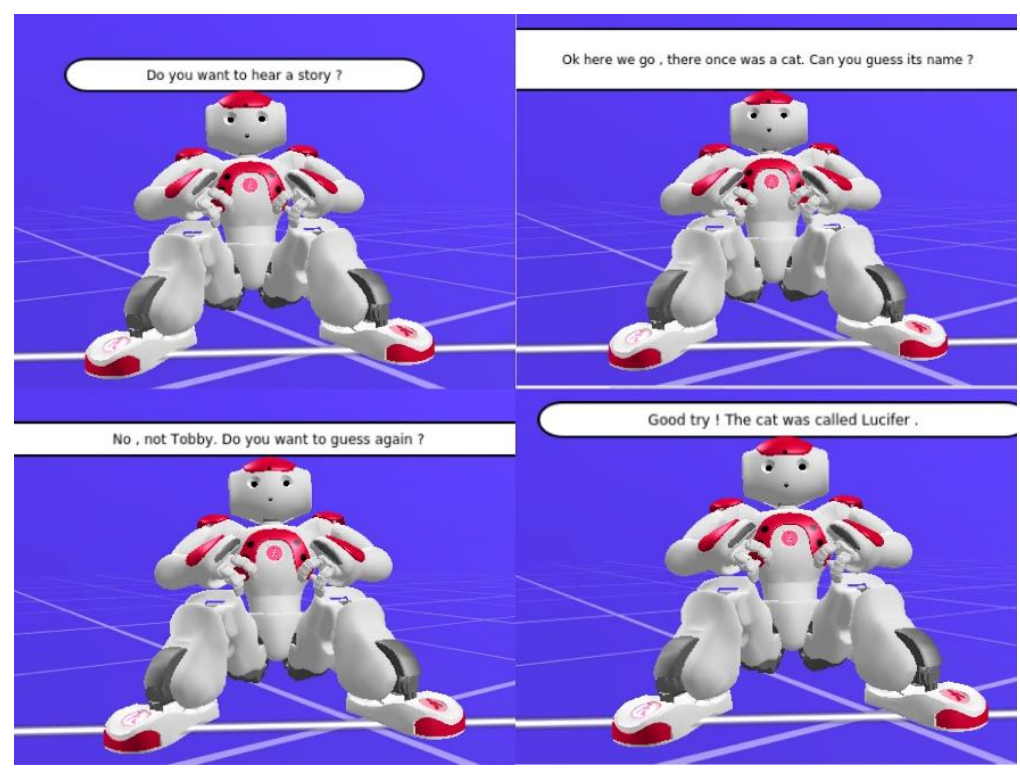

Fig. 3. Example of an interaction activity: Tell me a story. The NAO robot is not only trained on narration, it also actively invites the user to retrieve the context, guess the characters and what happens next in the plot. 


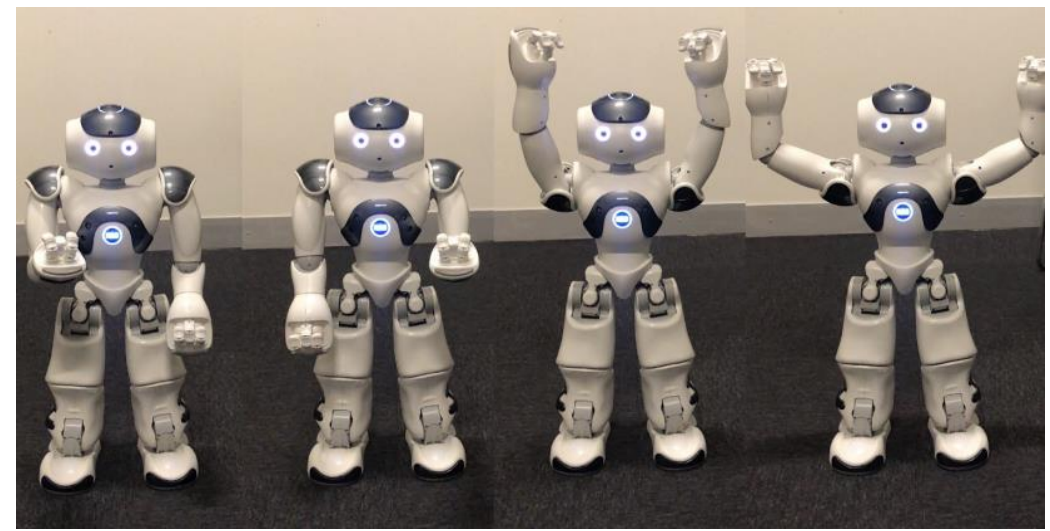

Fig. 4. Programming movement in NAO: Exercise routine. If the agent receives as input the "exercise option", it will perform a routine for the elderly to follow along. In between the exercise, the robot verbally interacts with the user, encouraging them to repeat the same movements, ask them if they feel well with it and/or cease the routine if the user chooses to.

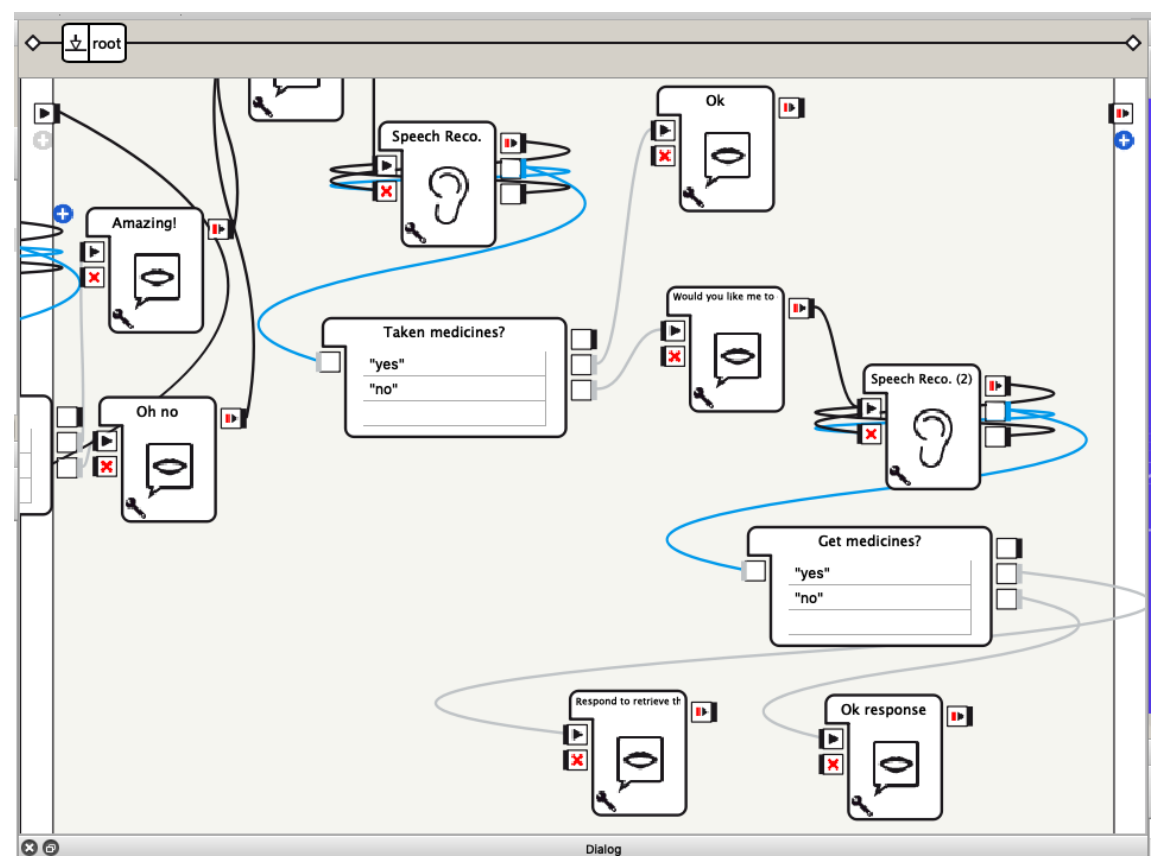

Fig. 5. Health assistant activity: Medication intake. NAO uses reminders to manage the medication intake of the elder. It starts by querying them if they have had their medicine and takes further assistive actions: reminding, describing or pointing the right medicine for the less able senior. 


\section{Conclusions}

In this work, we use the humanoid social robot NAO to design natural verbal and nonverbal interactions with a potential elder user, to grant personable assistance in their quotidian living. We programmed the agent using simple customisable algorithms to address two main activities: (a) disease management, helping the users with the daily intake of their medicines, setting reminders and selecting the right medicine box for the less able; (b) entertainment, to combat loneliness, support their physical and mental well-being through educational health context and interface the senior with digital technology. The carefully controlled laboratory-based trials surmise a promising behaviour of such social agents in supporting independent ageing in place.

\section{Acknowledgement}

Authors of this paper acknowledge the funding provided by the Interreg 2 Seas Mers Zeeën AGE'In project (2S05-014) to support the work described in this publication.

\section{References}

1. Bartels, S., Naslund, J. The Underside of the Silver Tsunami - Older Adults and Mental Health Care. The New England journal of medicine. 368. 10.1056/NEJMp1211456. (2013)

2. Netuveli, G., Blane, D., Quality of life in older ages, British Medical Bulletin, Volume 85, Issue 1, pp 113-126, https://doi.org/10.1093/bmb/ldn003 (2008)

3. Rabbitt, S. M., Kazdin A. E., Scassellati B., Integrating socially assistive robotics into mental healthcare interventions: Applications and recommendations for expanded use, Clinical Psychology Review 35, pp. 35-46 (2015)

4. Broadbent, E., Stafford, R., \& MacDonald, B. Acceptance of healthcare robots for the older population: Review and future directions. International Journal of Social Robotics, 1 (4), 319-330. doi:10.1007/s12369-009-0030-6 (2009)

5. Keay, A. \& Silicon Valley Robotics. Catalia health uses social robots to improve health outcomes. Robohub. From http://robohub.org/tag/silicon-valley-robotics/ (2017)

6. Agrigoroaie, R. M., \& Tapus, A. Developing a healthcare robot with personalized behaviors and social skills for the elderly, at the ACM International Conference on Human-Robot Interaction, Christchurch, New Zealand (pp. 589-590). New Jersey: IEEE Press (2016)

7. Molestina, K. UTA researchers using Shakespeare \& robots to help seniors. CBS Dallas Fort Worth. Retrieved fromhttp://dfw.cbslocal.com/ (2017)

8. O'Keeffe, D. Robot 'Matilda' helps engage older people living with dementia, new research shows. Australian Aging Agenda. http://www.australianageingagenda.com.au/ (2017)

9. Roger, K., Guse, L., Mordoch, E., Osterreicher, A. Social commitment robots and dementia. Canadian Journal on Aging, pp 87-94. doi:10.1017/S0714980811000663 (2012)

10. Shen, Z., \& Wu, Y. (2016). Investigation of practical use of humanoid robots in elderly care centres. Paper presented at the ACM International Conference on Human Agent Interaction (pp. 63-66). Biopolis, Singapore. doi:10.1145/2974804.2980485

11. Kumahara, Y., \& Mori, Y. Portable robot inspiring walking in elderly people. Paper presented at the ACM International Conference on Human Agent Interaction (pp. 145-148). Tsukuba, Japan. doi:10.1145/2658861.2658908 (2014) 
12. Wada, K., \& Shibata, T. Living with seal robots-Its sociophysical and physiological influences on the elderly at a care house. IEEE Transactions on Robotics, 23(5), 972-980. doi:10.1109/TRO.2007.906261 (2007)

13. Tamura, T., Yonemitsu, S., Itoh, A., Oikawa, D., Kawakami, A., Higashi, Y., Nakajima, K. Is an entertainment robot useful in the care of elderly people with severe dementia? The Journals of Gerontology Series A: Biological Sciences and Medical Sciences, 59 (1), M83M85 (2004)

14. Kramer, S. C., Friedmann, E., \& Bernstein, P. L. Comparison of the effect of human interaction, animal-assisted therapy, and AIBO assisted therapy on long-term care residents with dementia. Anthrozoös, 22 (1), 43-57. doi:10.2752/175303708X390464 (2009)

15. Khosla, R., \& Chu, M. Embodying care in Matilda: An affective communication robot for emotional wellbeing of older people Australian residential care facilities. ACM Transactions on Management Information Systems, 4(4), 1-33 (2013)

16. Khosla, R., Chu, M., Kachouie, R., Yamada, K., Yoshihiro, F., \& Yamaguchi, T. Interactive multimodal social robot for improving quality of care of elderly in Australian nursing homes. ACM International Conference on Multimedia (1173-1176). Nara, Japan (2012)

17. Mordoch, E., Osterreicher, A., Guse, L., Roger, K., \& Thompson, G. Use of social commitment robots in the care of elderly people with dementia: A literature review. Maturitas, 74, 14-20. doi:10.1016/j.maturitas.2012.10.015 (2012)

18. Lammer, L., Huber, A., Weiss, A., \& Vincze, M. Mutual care: How older adults react when they should help their care robot. Proceedings of the 3rd international symposium on New Frontiers in Human-Robot interaction, London, England (2014)

19. Louie, W. Y., McColl, D., \& Nejat, G. Acceptance and attitudes toward a human-like socially assistive robot by older adults. Assistive Technology: The Official Journal of RESNA, 26 (3), 140-150. doi:10.1080/10400435.2013.869703 (2014)

20. McEvoy, P., \& Plant, R. Dementia care: Using empathic curiosity to establish the common ground that is necessary for meaningful communication. Journal of Psychiatric and Mental Health Nursing, 21 (6), 477-482 (2014)

21. Nunez, E., Matsuda, S., Hirokawa, M., \& Suzuki, K. Humanoid robot assisted training for facial expressions recognition based on affective feedback, at the International Conference on Social Robotics, Paris, France. Shah, Wiken, Williams \& Breazeal (2015)

22. Romeo, M, Hernandez Garcia, D, Jones, R \& Cangelosi, A. Deploying a Deep Learning Agent for HRI with Potential "end-users" at Multiple Sheltered Housing Sites. in 7th International Conference on Human-Agent Interaction, Kyoto, Japan (2019)

23. Luperto, M, Romeo, M, Lunardini, F, Basilico, N, Abbate, C, Jones, R, Cangelosi, A, Ferrante, S \& Borghese, NA, Evaluating the Acceptability of Assistive Robots for Early Detection of Mild Cognitive Impairment. in 2019 IEEE/RSJ International Conference on Intelligent Robots and Systems, Macao, China, (2019)

24. Johnson, D. O., Cuijpers, R. H., Juola, J. F., Torta, E., Simonov, M., Frisiello, A., Beck, C. Socially assistive robots: A comprehensive approach to extending independent living. International Journal of Social Robotics, 6, 195-211. doi:10.1007/s12369-0130217-8 (2014)

25. Tanaka, M., Ishii, A., Yamano, E., Ogikubo, H., Okazaki, M., Kamimura, K., Watanabe, Y. Effect of a human-type communication robot on cognitive function in elderly women living alone. Medical Science Monitor: International Medical Journal of Experimental and Clinical Research, 18 (9), CR550-CR557. doi:10.12659/MSM.883350 (2012)

26. SoftBank Robotics Webpage https://www.softbankrobotics.com/emea/en/nao

27. SoftBank Robotics https://www.softbankrobotics.com/emea/en/industries/healthcare 